\title{
Encoding of Intention and Spatial Location in the Posterior Parietal Cortex
}

The posterior parietal cortex is functionally situated between sensory cortex and motor cortex. The responses of cells in this area are ditficult to classity as strictly sensory or motor, since many have both sensory- and movement-related activities, as well as activities related to higher cognitive functions such as attention and intention. In this review we will provide evidence that the posterior parietal cortex is an interface between sensory and motor structures end performs various functions important for sensory-motor integration. The review will focus on two specific sensory-motor tasks - the formation of motor plans and the abstract representation of space. Colls in the lateral intraparietal area, a subdivision of the parietal cortex, have activity related to eye movements the animal intends to make. This finding represents the lowest stage in the sensory-motor cortical pathway in which activity related to intention has been found and may represent the cortical stage in which sensory signals go "over the hump" to become intentions and plans to make movements. The second part of the review will discuss the representation of spece in the posterior parietal cortex. Encoding spetial locations is an essential step in sensor -motor transformations. Since movements are made to locations in space, these locations should be coded invarient of eye and head position or the sensory modality signaling the target for a movement. Data will be reviewed demonstrating that there exists in the posterior parietal cortex an abstract representation of space that is constructed from the integration of visual, auditory, vestibular, eye position, and proprioceptive head position signals. This representation is in the form of a population code and the above signals are not combined in a hephazard feshion. Rather, they are brought together using a specific operation to form "planar gain fields" that are the common foundation of the population code for the neural construct of space.

The first half of this review will show that the posterior parietal cortex is intimately involved in the process of intention. At some point in the sensory-motor pathway sensory signals give way to signals related to what the animal intends to do. This step from sensory representation to making a decision to perform a movement utilizes the neural circuitry within the posterior parietal cortex. The study of this transition from sensation to intention has been derived from the recent discovery of a small area within the posterior parietal cortex, the lateral intraparietal area (LIP), which is specialized for saccadic eye movements. This area analyzes sensory information related to the targets for saccades as well as conveying signals related to the plans to make eye movements.

The second half of this review will discuss recent neurophysiological experiments indicating that there exists in the posterior parietal cortex intermediate and abstract representations of space interposed between sensory input and motor output. Visual signals are combined with eye and head position signals to represent locations of visual targets in head, body and potentially even world-centered coordinates. Evidence is now emerging that indicates that auditory signals also contribute to this spatial representation and that the visual and auditory modalities are brought into the same coordinate frame in this area.
Richard A. Andersen

Division of Biology, California Institute of Technology, Pasadena, California 91125

\section{Posterior Eye Fiolds}

It has been appreciated for some time that the posterior parietal cortex plays a direct role in eye movements. Human patients with bilateral lesions to the posterior parietal cortex are unable to make willed saccades, although spontaneous saccades are unaffected (Balint, 1909). Electrical stimulation of monkey posterior parietal cortex results in saccadic eye movements (Fleming and Crosby, 1955; Wagman, 1964) and lesions in the monkey also produce deficits in saccades (Keating and Gooley, 1988; Lynch and McLaren, 1989).

In anatomical experiments investigating the connections of the posterior parietal cortex we injected retrograde tracers into the frontal eye fields and adjoining dorsolateral prefrontal cortex. Label was found predominantly within the lateral bank of the intraparietal cortex (Andersen et al., 1985a). We named this area the lateral intraparietal area (LIP) because of its location on the lateral bank of the intraparietal sulcus, lateral to the ventral intraparietal area (VIP; Maunsell and Van Essen, 1983; Fig. 1). Later experiments by our laboratory showed that most area LIP cells (63\%) had activity related to saccades, with the majority of them responding prior to saccades (Gnadt and Andersen, 1988; Andersen et al., 1990a; Barash et al., 1991a,b).

Area LIP is not the only region in the posterior parietal cortex showing a high degree of anatomical and functional specialization. Studies from other laboratories have shown that smooth pursuit activity is found mostly in area MST (Newsome et al., 1988), while reach activity is restricted largely to area $7 \mathrm{~b}$ (Hyvärinen and Shelepin, 1979; Robinson and Burton, 1980a,b; Hyvärinen, 1981), and eye position activity is found primarily in areas 7a and LIP (Lynch et al., 1977; Sakata et al., 1980; Andersen et al., 1987, 1990b; Andersen, 1989; Fig. 1). Recently a second eye field (medial parietal eye field, MP) has been discovered by our group (Thier and Andersen, 1993) that is located on the medial surface of the cerebral hemisphere, within cytoarchitectural area PGm of the posterior parietal cortex.

Thus, the posterior parietal cortex is composed of many functionally distinct subdivisions. There are two posterior eye fields in this area that are specialized for saccades, areas LIP and MP.

\section{Visual and Saccade-Related Activity of LP Neurons}

In the earty period of neurophysiological study of the posterior parietal cortex there was debate about whether parietal cortex was best considered a sensory or a motor area. When Mountcastle et al. (1975) first observed saccade responses in this area, they proposed that these responses were general commands for saccades. Robinson et al. (1978) then observed visual responses from neurons in the posterior parietal cortex. They challenged Mountcastle's command hypothesis, believing that the cells were giving sensory-related activities rather than movement related activities to the saccade targets. Andersen et al. (1987) later showed that posterior parietal neurons had both visual and saccade-related activity using a mem- 
A

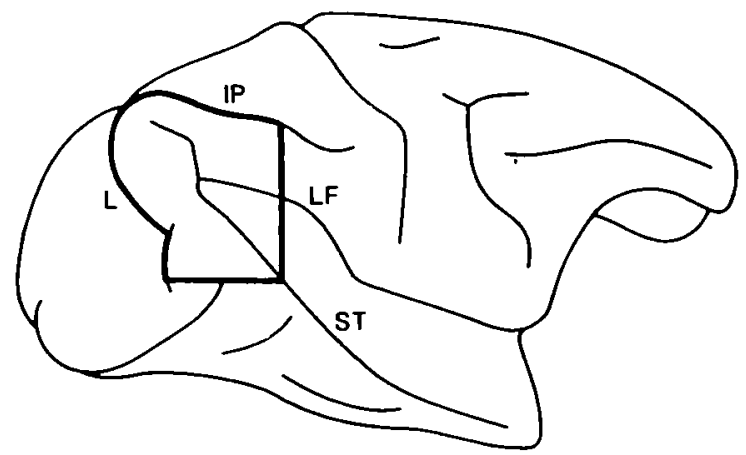

$1 \mathrm{~cm}$

C

6

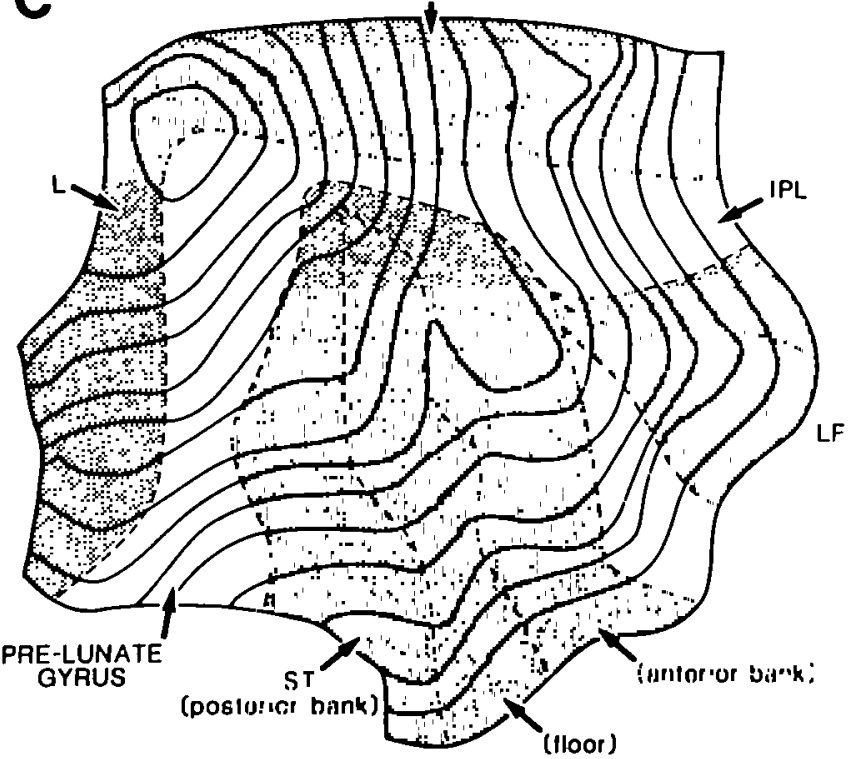

B
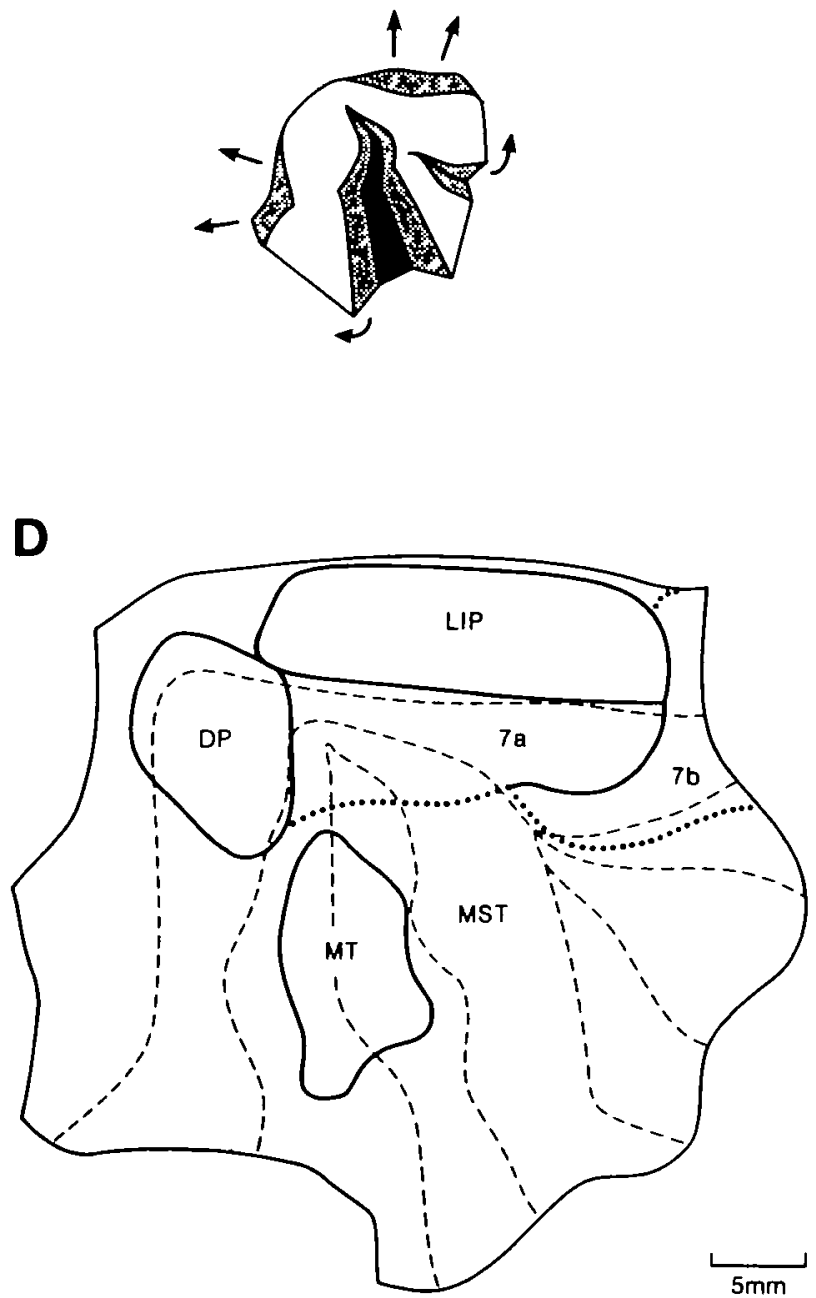

Figure 1. Parcellation of inferior parietal lobule and adjoining dorsal aspect of the prelunate gyrus used in this study. The cortical areas are represented on flattened reconstructions of the cortex. A Lateral view of the monkey hemisphere. The darker line indicates the area to be flattened. B, The same cortex isolated from the rest of the brein. The stippled areas are cortex buried in sulci, and the blackened ares is the floor of the superior temporal sulcus. The amows indicate movement of local cortical regions resulting from the mechanical flattening. $C$. The completehy flattened representation of the same area. The stappled areas represent cortical regions buried in sulci, and the contourlike lines are tracings of layer $N$ taken from frontal sections through this area. $D$, Locations of several of the cortical areas. The dashed lines indicate borders of cortical fields that are not precisely determinabłe. From Andersen (1987).

ory saccade task that separated sensory from motor responses. These results suggested that a better interpretation would be that the posterior parietal cortex is involved in sensorimotor integration, having both sensory and motor activities (Andersen, 1987).

We have recently studied the visual and saccade activity in LIP in greater detail and compared it to those activities in area 7a (Barash et al., 1991a,b). We found that saccade-related activity in area LIP typically begins prior to eye movements, unlike area $7 \mathrm{a}$, where most saccade responses begin after the initiation of eye movements. The visual receptive ficlds and motor fields of LIP neurons are usually found to overlap. The memory-related activity in memory saccade tasks is typically a more prominent feature of area LIP neurons than area $7 \mathrm{a}$ neurons. Since this memory activity of many cells codes the plan to make a movement, this result and the saccade latency result led us to propose that area LIP participates in the processing of saccades, whereas area 7 a likely performs other functions.

\section{Intended Movement Activity}

We have tested memory-related activity in area LIP in a delay task in which monkeys are required to make saccades to remembered locations in the dark (Gnadt and Andersen, 1988; Andersen et al., 1990a; Barash et al., 1991a,b). The LIP cells were found to remain active during the interval in which the animal was required to withhold its response while remembering the location of an extinguished saccade target (Fig. 2). To distinguish between the possibility that the cells were either coding the location of the sensory stimulus or coding the intention to make a saccade of a particular amplitude and direction, we used a double saccade task similar to the one developed by Mays and Sparks (1980). Our result was that activity could be evoked even when the sensory stimulus did not fall in the receptive field as long as the eye movement was made into the cell's motor field, suggesting that intention to make a motor movement was a critical feature (Fig. 3; Gnadt and Andersen, 1988). The activity appears to us to be part of a motor plan that has been locked in, and then remains active during the waiting period. We later found that the 
A) $200 \mathrm{msec}$ Delay

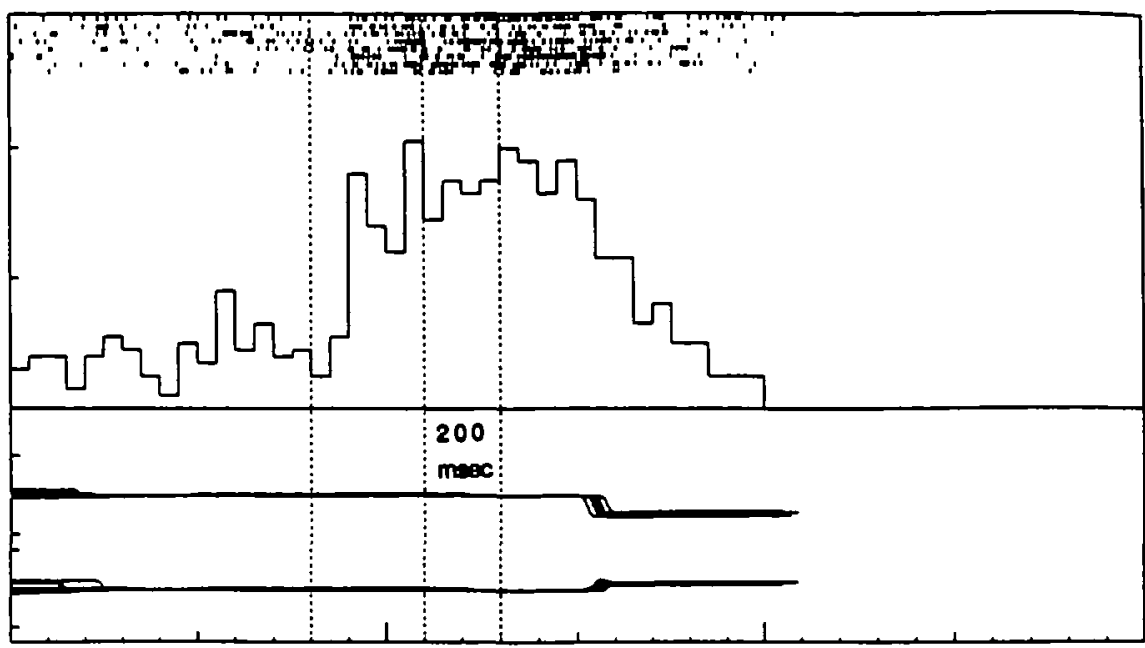

B) 1000 msec Delay

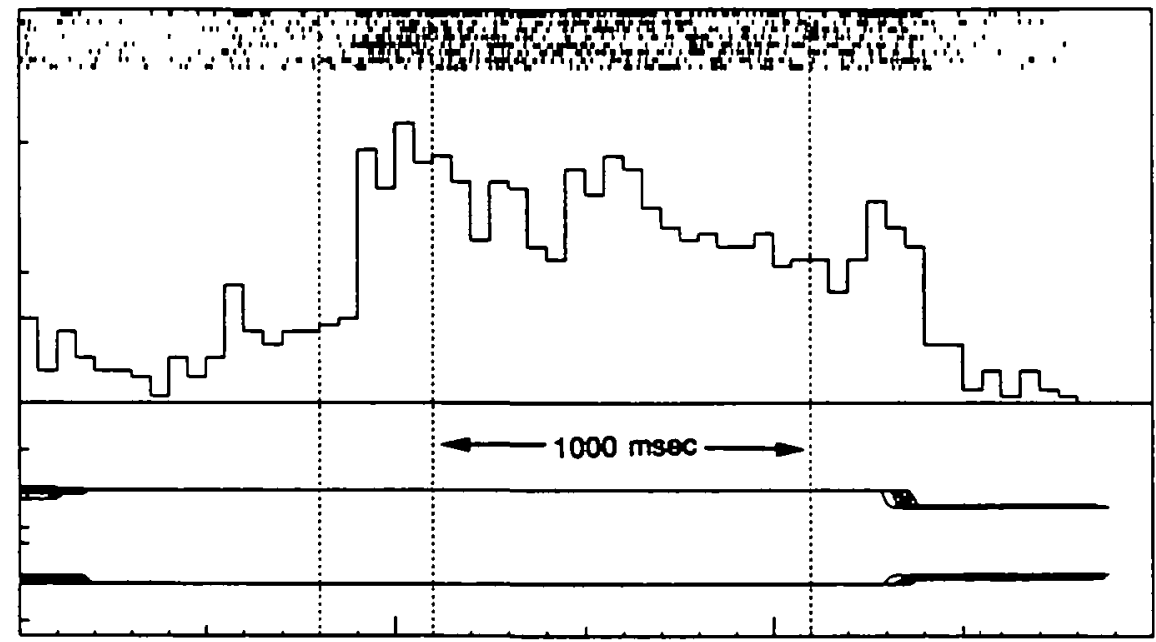

\section{C) 1300 msec Delay}

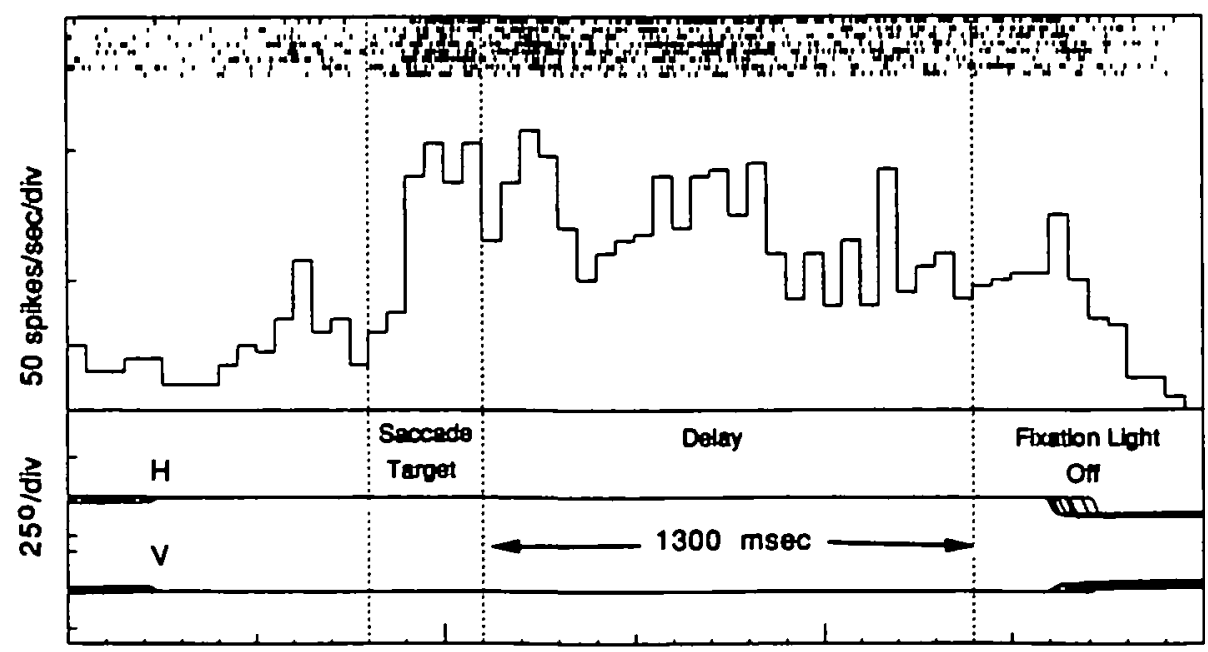

$100 \mathrm{msecs} / \mathrm{div}$
Figure 2. Memory saccade task with different delays demonstrating the memory charecter of the activity during the delay. Delays are $2000 \mathrm{msec}(A), 1000 \mathrm{msec}$ (B) and $1300 \mathrm{msec}(C)$. The rasters show the actual neural activity that is used to make the histograms. The period be tween the first two dotted vertical lines represents the time the seccede target is present and the period between the second and third fines is the delay period. The fixation light goes oft coincident with the third dotted vertical line. Both horizontal $(h)$ and vertical ( $h$ eye position traces are shown. In this experiment the saccade target appeared $15^{\circ}$ to the left There is a vertical component in the leftward eye movement; this upward component for horizontal eye movernents is common for saccades to remembered locations made in the dark From Andersen et al. (1990). 

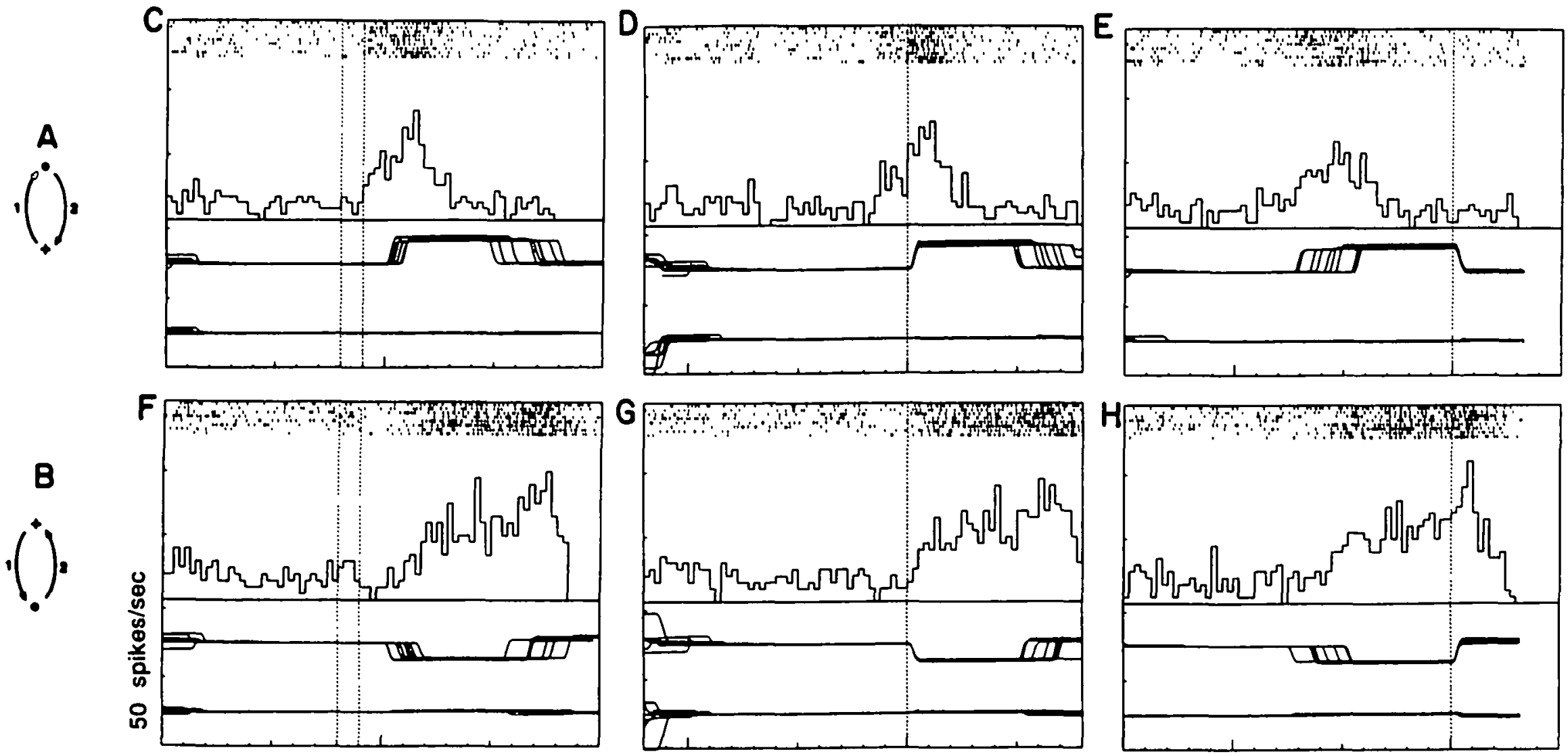

Figure 3. Back-saccade paradigm. $A$ and $B$, Scheme of the two saccades in the task. The first saccade is to the (single) terget, the second saccade is made in the dark back to the location of the original fixation point. C-H, Activity in the back-saccade task of an LIP neuron. The preferred direction of this neuron, for the $L S, M$, and $S$ phases, is upward. Hence, in the top row, the visual stimulation and the first movement are in the preferred direction, and the second movernent is in the opposite, nonpreferred direction. In the bottom row the visual stimulation and the first saccade are in the nonpreferred direction, but the second saccade is in the preferred direction. $C$ and $F$ are aligned on the sensory stimuli. The first dotted vertical line denotes the offset of the fixation spot and the simultaneous onset of the target The second dotted line represents the target offset. $D$ and $G$ are aligned on the beginning of the first seccede, and the dotted line denotes the time the first saccade begins. $E$ and $H$ are aligned on the beginning of the second saccade, and the dotted line denotes the tame the second saccade begins. Shown in each panel are, at the top, the spike rasters, where each honzontal trace represents a tral, and each tick mark within a line marks the time of occurrence of a spike; middle, the resulting histogram; and bottom, the horizontal and vertical eye-position traces of the verious trials, superimposed. From Barash et al. (1991). 
memory activity of LIP cells is directionally tuned, and interestingly these memory fields coincide with the visual and saccade fields for individual neurons (Barash et al., 1991a,b). We have hypothesized that the memory activity of many cells reflects the intention of the monkey to make his next saccade.

To examine our hypothesis that this activity is related to intention, we have recently trained monkeys in a "change in plan" task (Bracewell et al., 1991). Similar to previous memory-saccade tasks, the animal is required to make an eye movement to a remembered target after a delay. During the delay period of some trials, however, a second target is flashed at a new location, requiring a change in the direction of the planned saccade but still necessitating the animal to withhold the saccade until the end of the delay period. We found that the activity of the cells was consistent with the changes in motor plan required by the task - that is, they turned on and off with changes in intended targets. This was a most exciting result, as we found that the animal's plan could be determined by examining the activity of LIP cells without the animal demonstrating any overt behavior. To extend our examination of this intention idea we decided to train monkeys to make saccades to the remembered location of auditory targets (Bracewell et al., 1991; Mazzoni, 1994). We found that many of the cells demonstrated intended movement activity for both visual and auditory stimuli, supporting our idea that the cells activity is related more closely to the plan to make an eye movement than to the modality of the sensory stimulus. In a final memory double saccade task, we looked for memory activity and found that for many cells it was only present for the next intended movement (Bracewell, 1991; Bracewell et al., 1991; Mazzoni, 1992, 1994). In this particular task, two targets were flashed briefly; the animal was required to remember the location of both targets. The first target was flashed slightly before the second, and after the delay period the animal was required to make the first eye movement to the remembered earlier target location and then to make a second saccade toward the remembered location of the later target. If the second target fell within the visual receptive field of the cell, but the two saccades differed in amplitude and direction from the motor field of the test cell, then many of the cells tended to show no response. Even when the task was ordered such that the second saccade target fell into the visual receptive field and the second saccadic eye movement was made into the motor field, many of the cells persisted in not becoming active until after the first saccade.

These experiments argue strongly for the fact that activity during the delay period is related to intention for a large proportion of LIP neurons, because the activity just described signaled the direction of the impending movement and not the memory of the location of the visual stimuli. Of course it can be difficult to determine whether neural activity is related to attention or intention. Goldberg et al. (1990) suggest that such memory activity is related to the monkey's visual attention, rather than its intention to make movements. While either interpretation would be consistent with the "change in plan" results, it would not explain the auditory memory results. To account for the auditory results the idea of visual attention would have to be abandoned for a much broader definition of attention that was independent of modality. In our estimation, the results of the memory double saccade task do not appear to be consistent with a simple attention hypothesis. Despite the fact that the animal must attend to and memorize the location of both visual targets, many cells will nevertheless have little or no memory activity for the visual targets in their receptive fields if the task does not require eye movements into their motor fields (Bracewell et al., 1991; Mazzoni, 1994). A correlation of the memory activity with intention, then, and not with attention, seems to be the most satisfactory interpretation of these data.

In conclusion, area LIP appears to play a major role in the processing of saccadic eye movements. In particular, there is activity related to the intention to make eye movements, and this LIP activity appears to be the result of a transition from sensory signals to decisions to make eye movements.

\section{Representation of Space}

It is possible to form several intermediate representations of space by combining information from various modalities. The first such representation could be head-centered, which refers to a coordinate system framed with respect to the head. It would be formed by combining information about the location of a visual stimulus imaged on the retinas and the position of the eyes in the orbits (Fig. 4). A body-centered coordinate frame or representation would be more elaborate and achieved by combining information about head position with respect to the body with the previously mentioned eye and retinal position information (Fig. 4). An even more complex and general representation could be thought of as existing in world-centered coordinates (Fig. 4), which could be achieved by combining vestibular signals with retinal and eye position signals. There is now experimental evidence to suggest that the posterior parietal cortex contains and uses all these representations.

\section{Head-Centered Coordinates in Area $7 a$}

Lesions to the posterior parietal cortex in human and nonhuman primates produce profound spatial deficits. We have examined how space is represented in the posterior parietal cortex by recording from individual neurons and testing their spatial receptive field properties in behaving monkeys. For neurons to code in head-centered coordinates, one might expect that receptive fields would need to be found that would be anchored in head-centered, rather than retinal, coordinates. If this were the case, then each time the eyes would move, the cell's receptor field would have to shift locations a corresponding distance in the opposite direction on the retina in order to code the same location in head-centered coordinates.

After we undertook our early investigations of area $7 \mathrm{a}$ of the posterior parietal cortex, however, we discovered that locations in head-centered coordinates were encoded in an entirely different format than we had anticipated (Andersen and Mountcastle, 1983; Andersen et al., 1985b). The receptive fields of the neurons did not shift their retinal locations when eye position changed. Rather, the visual and eye position signals interacted with one another to form "gain fields" in which the amplitude of the visual response was modulated by eye position (Andersen et al., 1985b; Fig. 5). We termed a majority of the gain fields as "planar" because the amplitude of the response to stimulation of the same area of the retina varied linearly with horizontal and vertical eye position (Andersen et al., 1985b). Approximately two-thirds of the cells sampled in posterior parietal cortex demonstrated significant gain fields and approximately $80 \%$ of them were planar or had a significant planar component (Andersen et al., 1990a).

\section{Distributed Representation}

These early results demonstrated that spatial locations are not represented explicitly at a single-cell level using receptive fields in space. This is not to say, however, the location of a tanget in head-centered coordinates cannot be determined. In fact, it can still be easily determined if the activity of several area $7 \mathrm{a}$ neurons are taken together. The representation of space can be considered to be distributed in this area over many neurons. Figure 6 demonstrates why this representation 


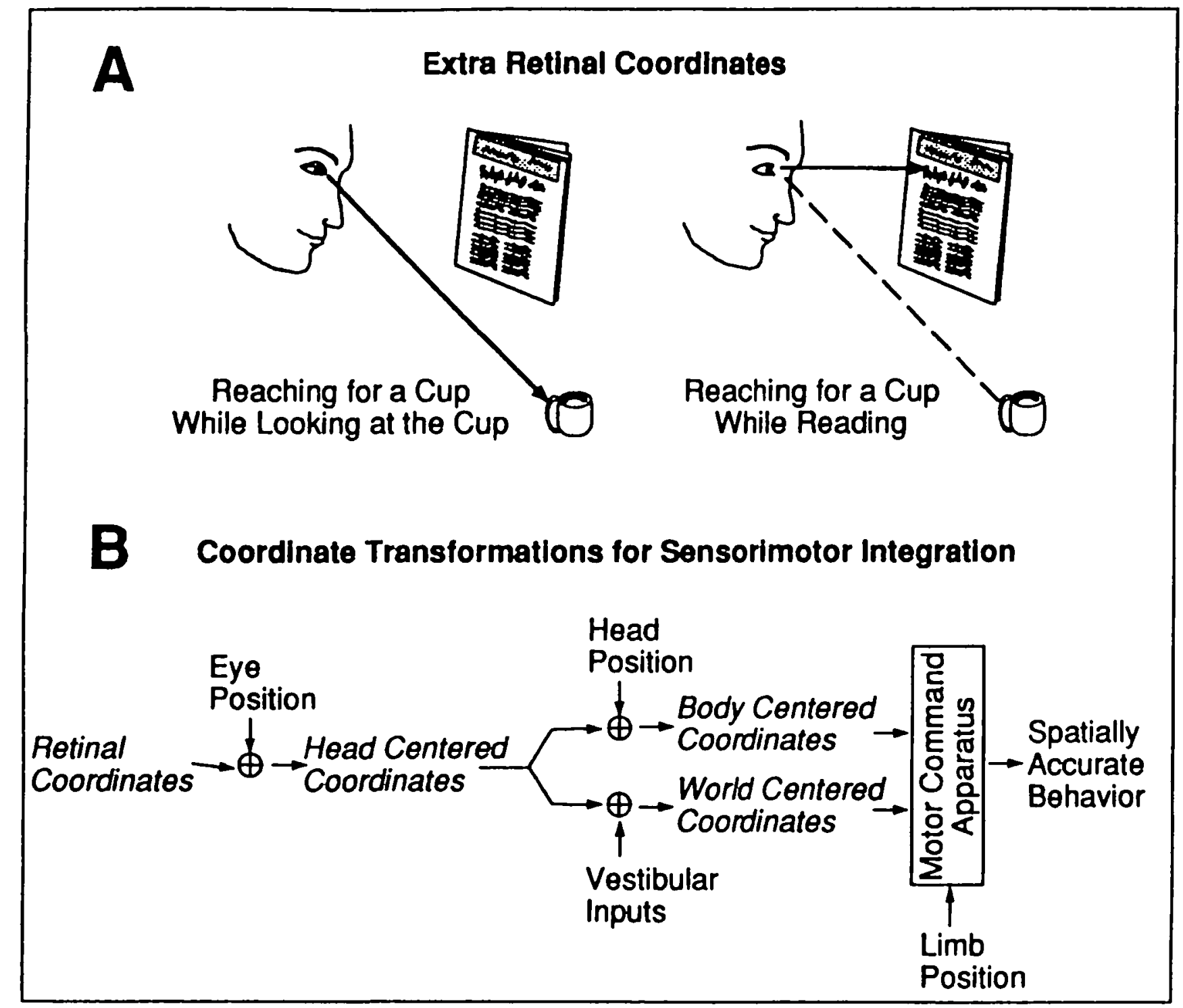

Figare 4. A, Demonstration of why representations of space in extraretinal coordinates are required for accurate motor behaviors. The term "extraratinal" refers to the encoding of visual stimuli in higher-level coordinate frames than simple retinal coordinates. In the sketch on the left a person is fixating the cup and it is imaged on the foveas, whereas on the right s/he is fixating the newrspaper and the cup is imaged on a peripheral part of the retinas. In both cases the subject is able to localize the cup with a reaching movement Since different parts of the retinas are stimulated in the two conditions, information about eye position must also be available to accurately determine that the cup was at the same locaton in spece. B. Schemetic showing how extraretinal coordinate frames can be computed from retinal coordinates. Eye position signals can be added to form representations in head-centered coordinates, and body-centered coordinates cen be formed by also adding head position information. One way of forming world coordinates is to add vestibular signals, which code the location of the head in the world, to a head-centered coordinate frame. The figure shows these signals being added sequentially for illustrative purposes. It is presently not known if there is a hierarchical organization of extraretinal coordinate frames in the brain, or if several of these signals come together at once to immediately form body- and world-coordinate frames, combined with information about limb position derived from proprioceptive inputs, to encode accurate reaching movements. From Andersen et al. (1993).

is distributed, using the contour plot of activity for the variables of location in head-centered space and eye position This plot illustrates that area 7 a neurons are tuned to a particular location in head-centered space, but only over a limited range of eye positions. The location that yields maximum response in head-centered coordinates can be thought of as the conjunction of the preferred eye position of the cell and the most responsive part of its retinal receptive field. Generating a signal, then, for location in head-centered space independent of eye position requires the activity of not one but a subset of parietal neurons. The code is thus a distributed one.

Distributed coding has been described in areas other than the parietal cortex. Area MT neurons, for example, are tuned to a limited range of temporal and spatial frequencies. A population of cells tuned to different temporal and spatial frequencies is required for stimuli of different shapes to be perceived as moving at the same speed. The perception of speed appears to use a distributed code not unlike the one for spatial location. The activity of single cells has come to be regarded as a component of a much larger, distributed network, an idea that is critical in advancing our understanding of how the brain computes locations in space.

\section{Mtcrostimultion Expertments}

Goodman and Andersen (1989) have examined eye movements produced by "microstimulating" the Zipser and Andersen (1988) model. The output of the neural network was con- 
a

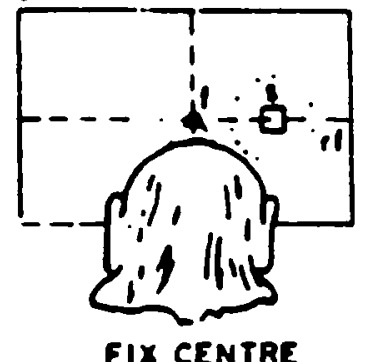

FIX CENTRE

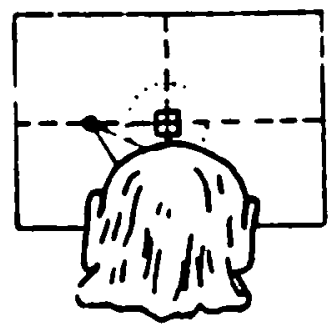

FIX LEFT $\boldsymbol{b}$
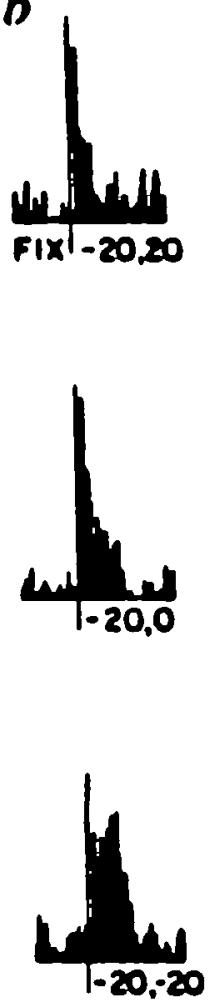
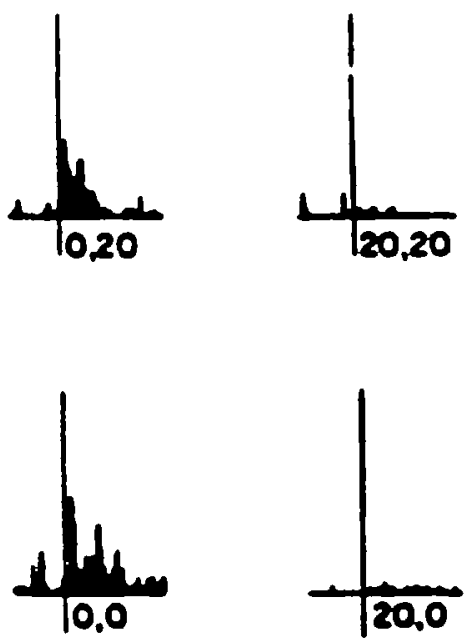

-

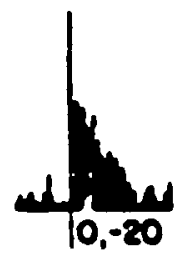

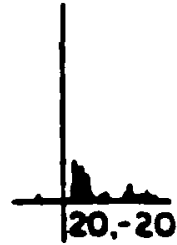

Finure 5. a, Expenmental protocol for determining spatial gain fields, with the projecton screen viewed from behind the monkey's head. To determine the effect of eye position, the monkey with head fixed, fixates on a point, $f$, at one of nine symmetrically placed locations on the projection screen. The stimulus, $S$, is always presented at the same retinal location, chosen as the maximum-response zone of the retinal-receptive field. The stimulus consists of spots of $1^{\circ}$ to $6^{\circ}$ diameter flashed for 500 msec. Each measurement is repeated eight times. b. Peristimulus histograms of a typical gain field determination. The nine histograms are located in the same relative positions as the fixations that produced them. The vertical line indicates the time of visual stimulus onset $c$, A graphic method for illustating these date in which the diameter of the darkened inner curcle, representing the visually evoked gain fields, is calculated by subtracting the background activity recorded $500 \mathrm{msec}$ before the stimulus onset from the total activity dunng the stimulus. The annulus diameter corresponds to the background activity that is due to an eye-position signal alone, recorded during the 500 msec before the stimulus presentation. From Zpper and Andersen (1988).

nected to a reduced set of oculomotor muscles (four instead of six). Microstimulation was produced by maximally activating individual hidden units. Stimulating the hidden units produced a change-in-amplitude pattern of eye movements similar to the pattern seen with electrical stimulation of area LIP in monkeys (Fig. $7 b$ ). In the change-in-amplitude pattern, the size of the saccade varies with eye position, but the direction of the saccade does not change. In Figure 7 the large dots represent the initial eye position prior to microstimulation, and the line represents the movement produced by stimulation.

The suggestion from this simulation is that the change-inamplitude pattern is indicative of a distributed representation of space in LIP. A single cell does not drive the eyes to a goal in space because the representation of head-centered space is distributed; rather, such behavior requires the activity of many LIP neurons. This idea was tested by simultaneously stimulating two or more hidden units in the model. Figure $7 a$ shows the results of stimulating two hidden units, which produces an eye movement toward a single goal, consistent with the distributed representation idea.

\section{Neural Networks}

Neural networks are computer models that we have trained to convert inputs of eye position and retinal position into locations in head coordinates at the output. Interestingly, we find they develop a distributed representation in their "hidden layer," which is interposed between the input and output layers (Zipser and Andersen, 1988). This distributed represen- tation in the computer model appears to be very similar to that found in area $7 a$; particularty notable is the fact that the "hidden" units exhibit planar gain fields. Mathematical analysis of the algorithm developed by the network suggests that the planar gain fields are the basis for adding eye and retinal position vectors in a distributed network (Goodman and Andersen, 1990; Brotchie et al., 1995). Such analyses support our earlier speculation that the method of integrating these two signals in the brain is not random, out rather requires the gain fields are planar.

We trained our neural network models for area 7 a to produce output units with receptive fields in head-centered coordinates (Zipser and Andersen, 1988). Once the network was trained the middle layer units were found to have gain fields similar to those found in area $7 \mathrm{a}$. These results suggested that gain fields are an intermediate step in the transformation from retinal to spatial receptive fields. A potential criticism of this network model is the fact that cells with receptive fields in space, the output of the model, are not routinely found. To make such an argument is to take the model too literally. The output representation using head-centered receptor fields simply shows that the information embedded in distributed form in the hidden layer can code location in head-centered space. The exact output format for reading out this information is not crucial and can be extracted in other brain structures, or in spatial behaviors, in a number of ways, For instance, we also trained networks with a monotonic output representation similar to the activity of neurons found in oc- 

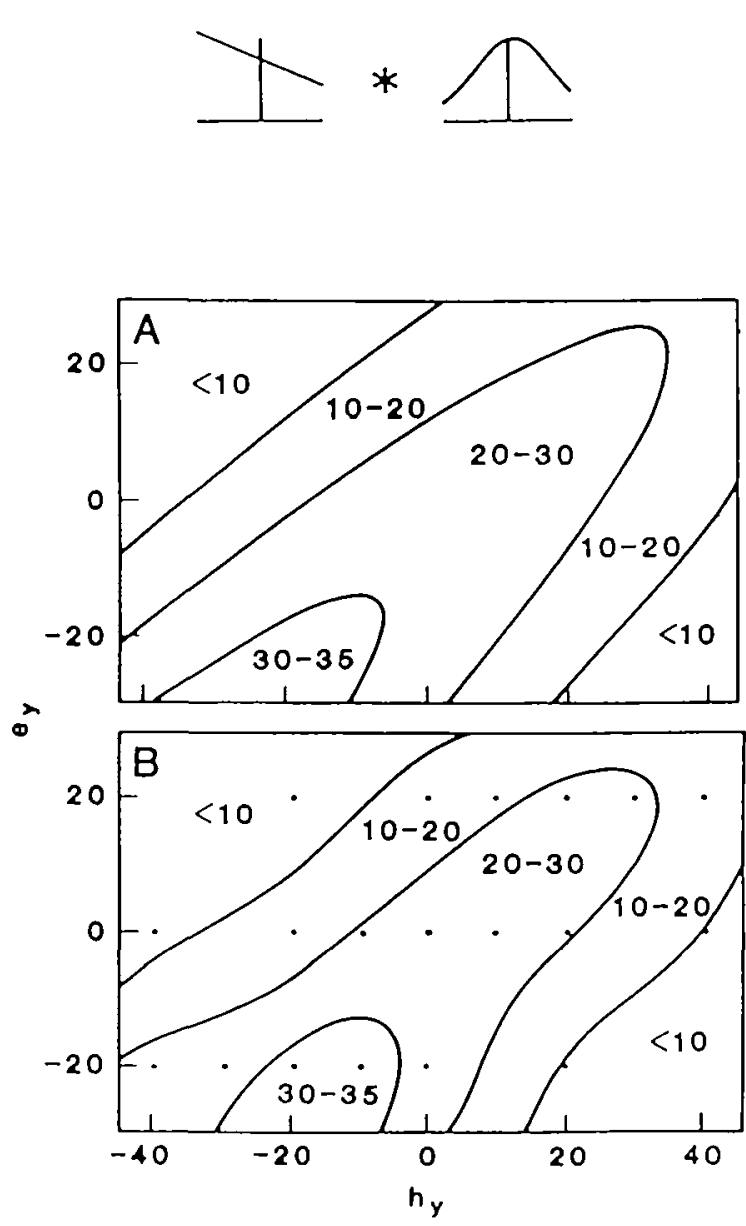

Figure 6. A, Computer simulation of the response (in spikes/sec) of an area 7a neuron predicted by multiptying the vertical axis of a planar gain field by the vertical axis of a Gaussian receptive field. The results are represented on the contour plot with the stimulus head-centered coordinates ( $h$ ) plotted along the abscissa and eye position $(\theta)$ along the ordinate. $B$, Contour plot of actual recording data for a cell with the same gain field and receptive field characteristics as the model neuron plotted in $A$. Each data point represents the mean evoked response to eight repetitions of the stimulus; the average standard error for these data points was 2 spikes/sec. From Andersen at al. (1985b).

ulomotor structures and motor centers in general. The activity of these output units varied linearty with location of the visual target referenced to the head. In a later simulation (Goodman and Andersen, 1989), we showed that such a network could be trained to make eye movements by using the output units to provide innervations to a set of extraocular muscles used to change the direction of gaze. This eye movement network also develops hidden units with planar gain fields. In light of these computer simulations, and the recording results, we have argued that receptive fields in space are an unnecessary encoding of spatial location (Goodman and Andersen, 1989; Andersen et al., 1990a). The brain can maintain representations of space in a distributed form and spatial receptive fields may be unnecessary for many spatial tasks.

Another potential criticism of the distributed representation idea involves the fact that area LIP projects to the superior colliculus (Asanuma et al., 1985; Lynch et al., 1985). Why convert retinal (oculocentric) signals to a representation of space in the posterior parietal cortex, only to reconvert it back to an oculocentric coordinate frame in the colliculus? One answer is that the posterior parietal cortex does not only project to the superior colliculus. One feature of the parietal coding format is that oculocentric information is not lost in the conversion to a distributed spatial representation, and can be recovered. For instance, if cells in the parietal cortex with the same retinal fields and all different directions of eye position tuning are converged onto cells in another structure, then the eye position signal is automatically subtracted out, leading to a recovery of the retinal vector. However, parietal cortex can also project to other areas, such as the frontal lobe and cerebellum where head- or body-centered information is important. A second interesting consideration comes from training the network using the colliculus as an output, but requiring the network to solve spatial problems. Krommenhoek et al. (1993) performed such a study, in which they trained a neural network to make double saccades in the Mays and Sparks (1980) paradigm. The output was to code in a collicular motor map the correct vector of each saccade. The network learned to make the correct second saccade, which required a spatial transformation by integrating the new eye position with the original retinal vector to generate a new, motor vector that would bring the eye to the correct position in space. Although there were no output cells with head-centered receptive fields, only collicular cells with oculocentric fields, the network developed gain fields similar to those found in the Zipser and Andersen (1988) model and the posterior parietal cortex. Thus, the network, when solving a spatial problem, develops an intrinsic representation of space in the hidden layer, even though the inputs and outputs are in eye-centered coordinates. Along a similar vein, Xing et al (1994) have trained a network to convert auditory and visual signals to a colliculus map at the output for making eye movements to both types of targets. The visual inputs are in retinal coordinates, the auditory inputs are in head-centered coordinates, and there is also an inputted eye position signal. Once this network has learned the task it also displays gain fields for the retinal visual targets. Interestingly, many of the hidden units also have auditory fields in retinal coordinates, and exhibit gain fields for the auditory signals. This result is very similar to the recording results of Stricanne et al. (1994) examining the coordinates of auditory activity in the posterior parietal cortex. Thus, these two models show that explicit receptive fields in space are not required for performing spatial tasks, and that the distributed form of spatial representation seems to be the optimal manner in which to represent space for many of these tasks.

It could also be argued that other factors such as attention modulate the activity of parietal cells, and thus would alter the spatial signal. In the distributed form of representation, it is likely that activating a subset of cells with receptive fields at a single location in the visual field would not change the output of the network and it would still code the correct spatial location. This is due to the distributed nature of the representation; increased activation of a particular retinal location would be balanced by the multitude of different directions of the gain field gradients at that location, and would not change the output.

Goldberg et al. (1990) have proposed a scheme by which the brain can always code in retinal coordinates, and could adjust for changes in eye position. This scheme uses a change in eye position vector (in retinal coordinates) that is subtracted from the current retinal position of a stimulus, to code a new retinal vector. This scheme has been applied to explain how the Mays and Sparks (1980) double eye movement task could be performed without resorting to a spatial representation. There would be two problems with applying this scheme to parietal cells. The first is that it is extremely limited, and is used to explain a single laboratory task, the double eye movement. It does not generalize well to the rich variety of spatial perceptions and behaviors of primates, such as reaching to targets, making head and eye movements to remem- 
(a)

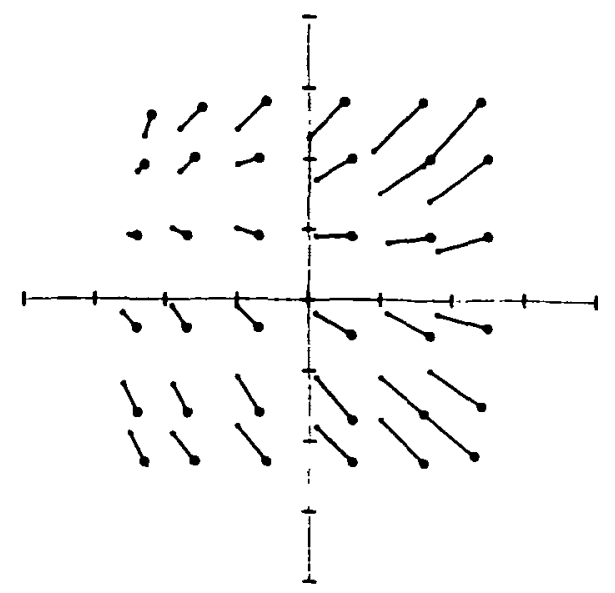

(b)
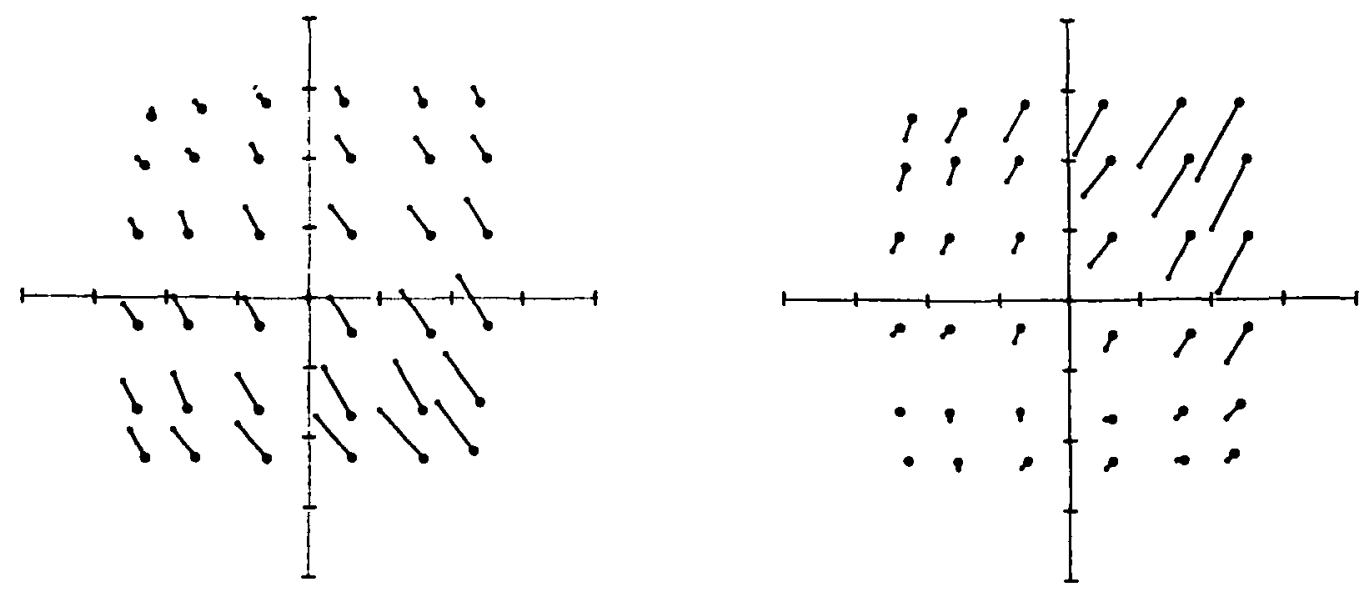

Figure 7. a, Eye movements recorded when two hidden units of a monotonic-output network are stimulated simultaneously. $b$, Eye movements recorded when each of the two hidden units is stimulated alone. Note that the result of simultaneous stimulation, illustrated in $a$, is more or less the vector addition of the two saccade fields that result from stimulation of the individual units. From Goodman and Andersen 1969).

bered locations in space, making eye movements to auditory targets, or navigating through space using vestibular cues. All of these behaviors can be easily performed using the distributed coding scheme (Goodman and Andersen, 1990; Xing et al., 1994). The second, and more problematic, consideration is that such a vector subtraction hypothesis requires that there be no eye position signals converging on the cells carrying the retinal vector signals. If there is such an input, the cells' activities will change for different initial eye positions and the activity to the same retinal vector stimulus would be different depending on the eye position. Thus, the cells would signal different retinal vectors for different eye positions, even though the retinal vector had not changed. Goldberg and Bruce (1990) realized this problem and showed one figure of a frontal eye field whose activity does not have a gain field for eye position. However, a majority of cells in the parietal lobe do have gain fields. It could still be argued that cye po- sition signals are subtracted out prior to the vertical subtraction. However, if parietal cortex were only coding information in retinal coordinates, it is difficult to understand why eye position signals are present, and require special mechanisms to then eliminate them.

\section{Gain Fields in Other Areas}

There have been several recent reports of gain fields in areas other than $7 \mathrm{a}$ of the posterior parietal cortex. These areas include cortical area LIP (Andersen et al., 1990a), cortical area V3a (Galletti and Battaglini, 1989), the inferior and lateral pulvinar (Robinson et al., 1990), and premotor and prefrontal cortex (Boussaoud et al., 1993) of monkeys. Cat area 17 (Weynand and Malpeli, 1993) has been found to have gain fields. These gain fields were usually found to be linear for horizontal and vertical eye positions when enough data were available to make such a determination. 
The finding that large numbers of areas have planar gain fields suggests this is the most predominant method of representing space and performing coordinate transformations. Interestingly, we found that networks with multiple hidden layers trained to make coordinate transformations have gain fields in all of the hidden layers (Mazzoni et al., 1991a). This result has parallels with the predominance of this form of representation in many parts of the brain, suggesting that once spatial information is compressed into the economical planar gain field format, it can be propagated throughout subsequent levels in the visual system.

There are also reports of receptive fields in space in some cortical areas (Fogassi et al., 1992; MacKay and Riehle, 1992; Battaglini et al., 1990; Graziano et al., 1995). It will be interesting to see if continued investigation will turn up more examples of spatial receptive fields, or if the distributed form will be the more common method for representing space.

\section{Distance}

So far we have only discussed representations with respect to the head in two dimensions-elevation and azimuth. Do parietal neurons also code in the third dimension of distance with respect to the head? Recent recording experiments by Gnadt and Mays suggest they do. They found LIP neurons that receive vergence angle signals that modulate the magnitude of the visually evoked responses. These cells are also tuned to disparity, and the disparity tuning does not change with vergence angle (Gnadt and Mays, 1991; Gnadt, 1992; Gnadt and Mays, 1995). Thus, there appear to be gain fields in the depth dimension as well. Interestingly, similar distance gain fields are predicted by neural network models similar to the Zipser and Andersen (1988) model, but trained to localize in depth (Lehky et al., 1990).

\section{Head Postion Gatn Fields}

Since our earlier experiments tested interactions of eye position and retinal position signals in monkeys with their heads mechanically immobilized, we could not distinguish between head- or body-referenced representations. Recently we have examined the effect of head position on visual responses with monkeys free to move their heads horizontally (Brotchie et al., 1995). We ran neural network simulations prior to these experiments, which predicted that cells representing space in body-centered coordinates should have gain fields for head and eye position, and these gain fields should be the same for individual cells. In other words, the gain fields should be a function of gaze position, independent of whether the eyes or head are used to direct gaze. Recording experiments from areas 7 $\mathbf{a}$ and LIP showed these predictions to be correct. Half of the cells with eye position gain fields also had similar head gain fields (approximately one-third of all cells sampled) These experiments raise the possibility that there may be two representations of space in the posterior parietal cortex, one in head-centered coordinates (using units with eye gain fields) and the other in body-centered coordinates (using units with both eye and head gain fields).

Recent recordings from our lab have examined the source of the head position signal. It could potentially be derived from efference copy of the animal's command to move the head. Efference copy, at least alone, does not appear to be a necessary source for the gain. We recorded the same gain fields in individual cells whether the animal points his head in different directions on his own or the head is mechanically fixed in these different directions. Proprioceptive signals are another possibility, and we have recently investigated two potential proprioceptive sources, neck proprioceptors and vestibular signals. Gain fields have been found in some cells when the animal's body is oriented in different directions and the head is maintained in a single direction; this result indicates that neck proprioception plays a role in some cells (Snyder and Andersen, 1994). When monkeys are rotated in a chair in the dark, many cells also demonstrate gain fields. In this condition gaze direction is changed by shifting the entire animal, and the head is always facing forward and the trunk and the eyes are always in the same orbital position (pointing straight ahead). This result suggests that vestibular signals can also produce gain fields. This modulation of the visual response by vestibular signals indicates that this population of cells may encode locations of visual stimuli in world coordinates.

Some cells also show gain fields derived from neck proprioception. In this case, the animal maintains its head in a single direction and the body is rotated under the neck. Many neurons demonstrate gain fields under these conditions.

\section{Auditory Signals}

An important question is how auditory signals might be brought into spatial register with visual signals in the posterior parietal cortex. We have recently examined this issue in monkeys trained to make memory saccades for briefly presented auditory targets in the dark (Mazzoni, 1994; Stricanne et al., 1994). Many area LIP cells are active during the memory period in this task. A majority of the cells have auditory fields that are in eye-centered coordinates and move with the eyes. Many of the responses also demonstrate planar gain fields. Since the visual, retinal receptive fields are also eye centered (the retina moves with the eyes), these results indicate that the auditory and visual signals use the same method, and coordinate system, for representation space in the posterior parietal cortex

\section{Btologically Plaustble Learnting Rule}

The major criticism of neural network models has been that the learning rule used for training the networks is unlikely to be used by the nervous system. To demonstrate that the gain field algorithm does not depend on the learning rule used to develop it (Mazzoni et al., 1991a,b) we trained a neural network to perform the transformation from retinal to head-centered coordinates using a more biologically plausible learning rule than backpropagation. This more plausible learning method is a variation of a reinforcement learning rule developed by Barto and Jordan (1987) that is more biologically plausible than the backpropagation rule we used in our original model (Fig. 8). The reinforcement trained networks produced planar gain fields similar to the backpropagation models and the recording data. The results from the reinforcement network strongly argue that the same algorithm for computing the coordinate transformation develops independent of the exact learning rule used to generate it. Similarly, posterior parietal neurons could learn or adjust spatial representations using planar gain fields that result from simple, biological learning mechanisms.

\section{Conclusions}

Area IJP plays a high-level role in the processing of eye movements. Its neurons integrate visual, auditory, eye position, and head position signals to represent targets in head- and bodycentered spatial coordinates or even world-centered coordinates. LIP also has memory-related activity that plays a role in the formation of motor plans. These results point to a central role for LIP for directing gaze.

The representation of space in the posterior parietal cortex is distributed. One issue for further research is whether there are different representations of space that are segregated on an anatomical basis. A proportion of cells show eye position effects only, whereas others show head position as well as 


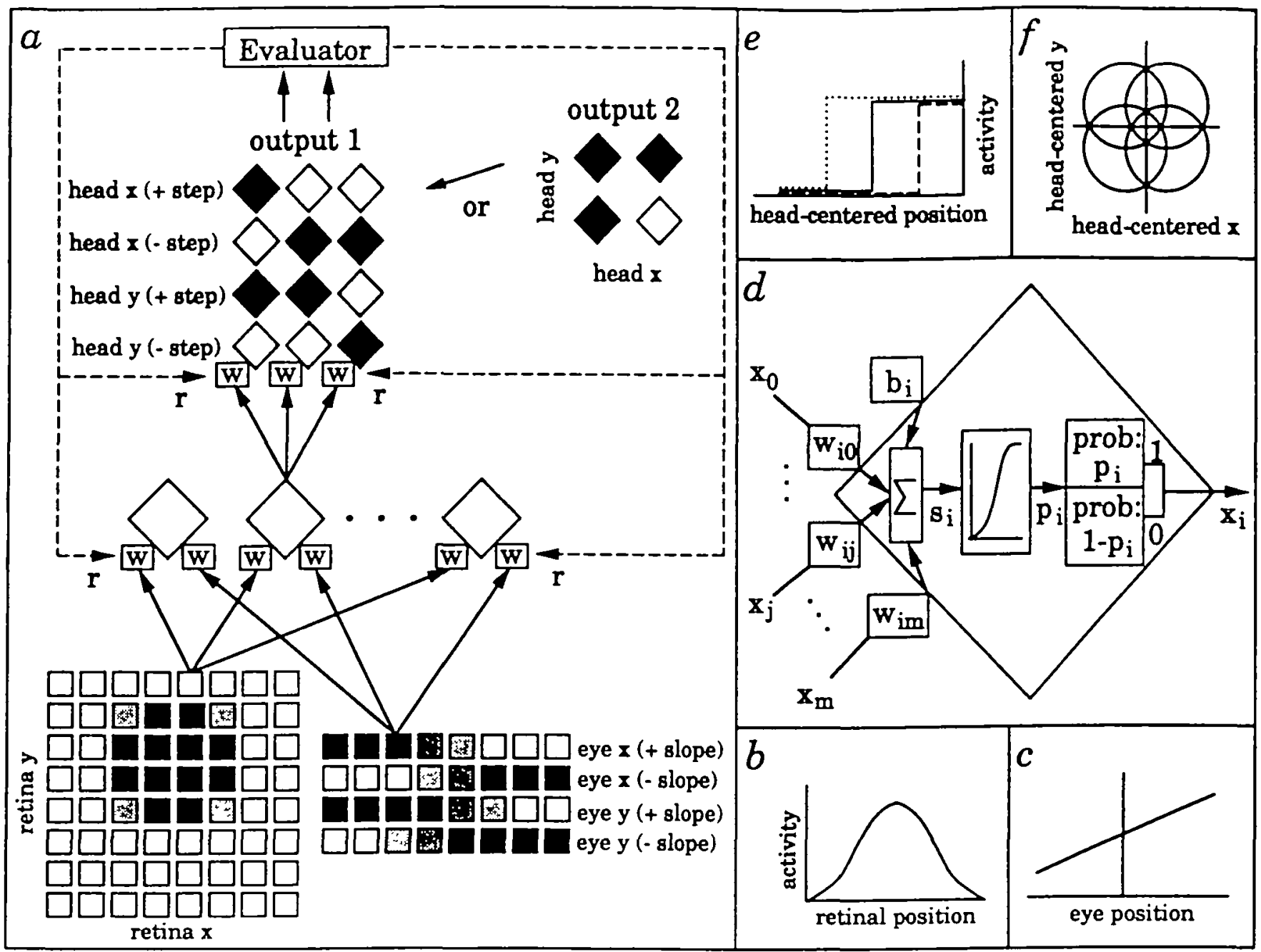

Figure 8. 8 , Network structure. $b$ and $c$, Retinal input is encoded by 64 units with Gaussien receptive fields $b$, while oye position is represented by 32 units with linear activation functions $c$. In the retinal input, each unit has an output between 0 and 1, a $1 / 6$ width of $15^{\circ}$, and a receptive fiald peak $10^{\circ}$ apart from that of its horizontal and vertical neighbors. In the eye-position input, the output of each unit, between 0 and 1 , is a linear function of horizontal or vertical orbital angle, with random slope and intercept These input formats reproduce properties of certain area 7a neurons that respond onty to visual stmuli or to changes in eye position. The shading of each unit is proportional to its activity, with black representing maximum actvity. $d$, The hidden end output layers are composed of binary stochastic elements, which produce an output of 1 with probability (prob) $p$ equal to the logistic function of the sum of the weighted inputs $\left(s_{1}=S_{i=1}, w_{i} x\right)$, and zero with probability $1-p$. The the unit in the network provides input $x_{j}$ to th unit via the connection $w_{i}$ $m$ is the number of inputs to the units, and $b$ is a bias. The network used from two to eight hidden units. The output units encode head-centered locations according to one of two output formats. In the "binary-monotonic" format (e), each unit produces an output of 1 or 0 , depending on whether the encoded locations is to the right or to the left lor, for some units, above or betow) a certain reference point For example, a typical output layer consisted of four sets of three units, giving an output of 1 when the $x$ (or $h$ craniotopic coordinate is $>$ lor $<1-40^{\circ}, 0$, or $+40^{\circ}$. This format is analogous to the eye-position input format, in that four groups of units encode an increase in horizontal or vertical position angle by increasing of decreasing their activation monotonically. Another format we used is the "binary-Gaussian" one ( $f$, in which four units give an output of 1 when the spatial position is within $100^{\circ}$ of their receptive field centers, which are located at $( \pm 60, \pm 60 \%$. This format is analogous to that of the retinal input, in that a position angle is encoded topographically by units with overlapping receptive fields. From Mazoni et al. (1991).

eye position effects. It is conceivable that these two populations represent two anatomically segregated representations of space, one in head-centered coordinates and the other in body-centered coordinates. Alternatively, if these two groups are mixed, then the population as a whole is perhaps coding in body-centered coordinates.

It is also still not known whether the coordinate transformations proceed in a hierarchical fashion. The body-centered cells of the posterior parietal cortex could be constructed by adding head position signals to the head-centered representation, or the entire representation could be body centered, with some cells exhibiting only retinal and eye position signals within this highly distributed representation (training networks to code in body-centered coordinates often produces a proportion of hidden units with only eye and retinal signals). The gain field idea can be extended to reference stimuli with respect to the arm by adding shoulder position signals. Are shoulder gain fields found in areas such as $7 \mathrm{~b}$ that have a convergence of joint angle and visual signals? Are there representations of visual space with respect to the hand? These and other issues should make the posterior parietal cortex a fruitful area for the study of higher brain functions for years to come.

\section{Notes}

I thank Larry Snyder for comments on a draft of the manuscript, and Laura Rodriguez and Carol Andersen for editorial assistance.

Address correspondence to Richard A. Andersen, Division of Biology 216-76, Californta Institute of Technology, Pasadena, CA 91125. 


\section{References}

Andersen RA (1987) The role of the inferior parietal lobule in spatial perception and visualmotor integration. In: The handbook of physiology, Sec 1, The nervous system, Vol V, Higher functions of the brain, Pt 2 (Plum F, Mountcastle VB, Geiger SR, eds), pp 483518. Bethesda, MD: American Physiological Society.

Andersen RA (1989) Visual and eye-movement functions of the posterior parietal cortex. Annu Rev Neurosci 12:377-403.

Andersen RA, Mountcastle VB (1983) The influence of the angle of gaze upon the excitability of the light sensitive neurons of the posterior parietal cortex. J Neurosci 3:532-548.

Andersen RA, Asanuma C, Cowan WM (1985a) Callosal and prefrontal associational projecting cell populations in area 72 of the macaque monkey: a study using retrogradely transported fluorescent dyes. J Comp Neurol 232:443-455.

Andersen RA, Essick GK, Siegel RM (1985b) Encoding of spatial location by posterior parietal neurons. Science 230:456-458.

Andersen RA, Essick GK, Siegel RM (1987) Neurons of area 7 activated by both visual stimuli and oculomotor behavior. Exp Brain Res 67:316-322.

Andersen RA, Bracewell RM, Barash S, Gnadt JW, Fogassi L (1990a) Eye position effects on visual, memory and saccade-related activity in areas LIP and 7a of macaque. J Neurosei 10:1176-1196.

Andersen RA, Asanuma C, Essick G, Siegel RM (1990b) Corticocortical connections of anatomically and physiologically defined subdivisions within the inferior parietal lobule. J Comp Neurol 296: 65-113.

Asanuma C, Andersen RA, Cowan WM (1985) The thalamic relations of the caudal inferior parietal lobule and the lateral prefrontal cortex in monkeys: divergent cortical projections from cell clusters in the medial pulvinar nucleus. J Comp Neurol 24:357-381.

Balint R (1909) Seelenlahmung des "Schauens," Optische Ataxie, Raumliche Storung der Aufmerksamkeit. Psychiatr Neurol 25:5181.

Barash S, Bracewell RM, Fogassi L, Gnadt JW, Andersen RA (1991a) Saccade-related activity in the lateral intraparietal area $I$. Temporal properties. J Neurophysiol 66:1095-1108.

Barash S, Bracewell RM, Fogassi L, Gnadt JW, Andersen RA (1991b) Saccade-related activity in the lateral intrapariental area. II. Spatial properties. J Neurophysiol 66:1109-1124.

Barto AG, Jordan MI (1987) Gradient following without backpropagation in layered networks. Proc IEEE Int Conf Neural Networks 2:629-636

Battaglini PP, Fattori P, Galletti C, Zeki S (1990) The physiology of area $V 6$ in the awake, behaving monkey. J Physiol (Lond) 423: $100 \mathrm{P}$.

Boussaoud D, Barth TM, W/se SP (1993) Effects of gaze on apparent visual responses of frontal cortex neurons. Exp Brain Res 93:423434

Bracewell RM, Barash S, Mazzoni P, Andersen RA (1991) Neurones in the macaque lateral intraparietal cortex (UP) appear to encode the next intended saccade. Soc Neurosci Abstr 17:1282

Bracewell RM (1991) On posterior parietal cortex and saccadic eye movements. PhD thesis, MIT.

Brotchic PR, Andersen RA, Snyder LH, Goodman SJ (1995) Head position signals used by parietal neurons to encode locations of visual stimuli. Nature 375:232-235.

Fleming JFR, Crosby EC (1955) The parietal lobe as an additional motor area: the motor effects of electrical stimulation and ablation of cortical areas 5 and 7 in monkeys. J Comp Neurol 103:485512.

Fogassi L, Gallese V, di Pellegrino G, Fadiga L, Gentilucci M, Luppino G, Matelli M, Pedotti A, Rizzolatte G (1992) Space coding by premotor cortex. Exp Brain Res 89:686-690

Galletti C, Battaglini PP (1989) Gaze-dependent visual neurons in area V3A of monkey prestriate cortex. J Neurosci 9:1112-1125

Gnadt JW (1992) Area LUP: three-dimensional space and visual to oculomotor transformation. Bchav Brain Sci 15:745-746.

Gnadt JW, Andersen RA (1988) Memory related motor planning actwity in posterior parietal cortex of macaque. Exp Brain Res 70 : 216-220.

Gnadt JW, Mays LE (1995) Neurons in monkey parietal area LIP are tuned for eye-movement parameters in three-dimensional space. J Neurophysiol 73:280-297.

Goldberg ME, Bruce CJ (1990) Primate frontal eye fields. ПI. Main- tenance of a spatially accurate saccade signal. J Neurophysiol 64 : 489-508.

Goldberg ME, Colby CL, Duhamel J-R (1990) The representation of visuomotor space in the parietal lobe of the monkey. Cold Spring Hartoor Symp Quant Biol 55:729-739.

Goodman S, Andersen RA (1989) Microstimulation of a neural-network model for visually guided saccades. J Cognit Neurosci 1: 317-326.

Goodman SJ, Andersen RA (1990) Algorithm programmed by a neural network model for coordinate transformation. Proc Int Joint Conf Neural Networks 2:381-386.

Graziano MSA, Yap GS, Gross CG (1995) Coding of visual space by premotor neurons. Science 266:1054-1057.

Hyvärinen J (1981) Regional distribution of functions in parietal association area 7 of the monkey. Brain Res 206:287-303.

Hyvärinen J, Shelepin Y (1979) Distribution of visual and somatıc functions in the parietal associative area 7 of the monkey. Brain Res 169:561-564.

Keating EG, Gooley SG (1988) Disconnection of parietal and occipital access to the saccade oculomotor system. Exp Brain Res 70: 385-398.

Krommenhoek KP, van Opstal AJ, Gielen CCAM, van Gisbergen JAM (1993) Remapping of neural activity in the motor colliculus; a neural network study. Vision Res 33:1287.

Lehky SR, Pouget A, Sejnowski TJ (1990) Neural models of binocular depth perception. Cold Spring Harbor Symp Quant Biol 55:765777.

Lynch JC, McLaren JW (1989) Deficits of visual attention and saccadic eye movements after lesions of parieto-occipital cortex in monkeys. J Neurophysiol 61:74-90.

Lynch JC, Mountcastle VB, Talbot WH, Yin TCT (1977) Parietal lobe mechanisms for directed visual attention.J Neurophysiol 40:362389.

Lynch JC, Graybiel AM, Lobeck IJ (1985) The differential projection of two cytoarchitectonic subdivisions of the inferior parietal lobule of macaque upon the deep layers of the superior colliculus. J Comp Neurol 235:241-254.

Mackay WA, Riehle A (1992) Planning a reach: spatial analysis by area 7 a neurons. In: Tutorials in motor behavior 2 (Stelmach G, Requin J, eds). New York: Elsevier.

Maunsell JHR, Van Essen DC (1983) The connections of the middle temporal visual area (MT) and their relationship to a cortical hierarchy in the macaque monkey. J Neurosci 3:2563-2586.

Mays LE, Sparks DL (1980) Dissociation of visual and saccade-related responses in superior colliculus neurons. Science 43:207-232.

Mazzoni P (1994) Spatial perception and movement planning in the posterior parietal cortex. PhD Thesis, MTT.

Mazzoni P, Andersen RA, Jordan MI (1991a) A more biologically plau sible learning rule for neural networks. Proc Natl Acad Sci USA 88:4433-4437.

Mazzoni P, Andersen RA, Jordan MI (1991b) A more biologically plausible learning rule than backpropagation applied to a network model of cortical area 7a. Cereb Cortex 1:293-307.

Mazzoni P, Bracewell RM, Barash S, Andersen R (1992) Intended movement activity of area LIP neurons in delayed double saccade tasks. Soc Neurosci Abstr 18:148.

Mountcastle VB, Lynch JC, Georgopoulos A, Sakata H, Acuna C (1975) Posterior parietal association cortex of the monkey: command function for operations within extrapersonal space. J Neurophy. siol 38:871-908.

Newsome WT, Wurtz. RH, Komatsu H (1988) Relation of cortical areas MT and MST to pursuit eye movements. I. Differentiation of retinal from extraretinal inputs. J Neurophysiol 60:604-620.

Robinson CJ, Burton H (19802) Organization of somatosensory receptive fields in cortical areas $7 \mathrm{~b}$, retroinsular postauditory and granular insula of $M$. fasctcularis. J Comp Neurol 192:69-92.

Robinson CJ, Burton H (1980b) Somatic submodality distribution within the second somatosensory (SII), 7b, retroinsular, postauditory and granular insular cortical areas of $M$. fascicularis. J Comp Neurol 192:93-108.

Robinson DL, Goldberg ME, Stanton GB (1978) Parietal association cortex in the primate: sensory mechanisms and behavioral modulations. J Neurophysiol 41.910-932.

Robinson DL, McClurkin JW, Kartzmann C (1990) Orbital position 
and eye movement influences on visual responses in the pulvinar nuclei of the behaving macaque. Exp Brain Res 82:235-246.

Sakata H, Shibutani H, Kawano K (1980) Spatial properties of visual fixation neurons in posterior parietal association cortex of the monkey. J Neurophysiol 43:1654-1672.

Snyder LH, Andersen RA (1994) Effects of vestibular and neck pro prioceptive signals on visual responses in posterior parietal cortex. Soc Neurosci Abstr 20:1278.

Stricanne B, Xing J, Mazzoni P, Andersen RA (1994) Response of LIP neurons to auditory targets for saccadic eye movements: a distributed coding for sensorimotor transformation. Soc Neurosci Abstr 20:143.

Thier P, Andersen RA (1993) Electrophysiological evidence for a second medial parietal eye field. Soc Neuroset Abstr 19:27.

Wagman IH (1964) Eye movements induced by electric stimulation of cerebrum in monkeys and their relationship to bodily movements. In: The oculomotor system. (Bender MB, ed), pp 18-39. New York: Harper \& Row.

Weynand TG, Malpeli JG (1993) Responses of neurons in primary visual cortex are modulated by eye position. J Neurophysiol 69 . 2258-2260.

Xing J, Stricanne B, Andersen RA (1994) A neural network model for sensorimotor transformation in macaque area LIP. Soc Neurosci Abstr 20:143.

Zjpser D, Andersen RA (1988) A back-propagation programmed network that simulates response properties of a subset of posterior parietal neurons. Nature 331:679-684. 